\title{
Proteasome granular localization is regulated through mitochondrial respiration and kinase signaling
}

\author{
Kenrick A Waite and Jeroen Roelofs"
}

6 Department of Biochemistry and Molecular Biology, University of Kansas Medical

7 Center, Kansas City, 3901 rainbow Blvd., HLSIC 1077,Kansas, USA

$8 \quad$ "correspondance: jroelofs@kumc.edu

9 Running title: Mitochondrial function regulates proteasome localization

11 Keywords: proteasome, liquid-liquid phase separation, mitochondrial respiration, MAP

12 kinases, proteasome storage granules (PSG), proteaphagy, mitochondrial inhibition,

13 autophagy. 


\section{Summary}

18 We determined a role for mitochondrial respiration in regulating proteasome granule

19 formation and identified the cell integrity MAP kinase pathway and Snf1 kinase as

20 regulatory factors.

22 Abstract

23 In yeast, proteasomes are enriched in cell nuclei where they execute important cellular

24 functions. Nutrient-stress can change this localization indicating proteasomes respond to

25 the cell's metabolic state. However, the signals that connect these processes remain

26 poorly understood. Carbon starvation triggers a reversible translocation of proteasomes

27 to cytosolic condensates known as proteasome storage granules (PSGs). Surprisingly,

28 we observed strongly reduced PSG levels when cells had active cellular respiration prior

29 to starvation. This suggests the mitochondrial activity of cells is a determining factor in

30 the response of proteasomes to carbon starvation. Consistent with this, upon inhibition of

31 mitochondrial function we observed proteasomes relocalize to granules. These links

32 between proteasomes and metabolism involve specific signaling pathways, as we

33 identified a MAP kinase cascade that is critical to the formation of proteasome granules

34 after respiratory growth but not following glycolytic growth. Furthermore, the yeast

35 homolog of AMP kinase, Snf1, is important for proteasome granule formation induced by

36 mitochondrial inhibitors, while dispensable for granule formation following carbon

37 starvation. We propose a model where mitochondrial activity promotes proteasome 38 nuclear localization. 


\section{Introduction}

The efficiency of many cellular processes relies on optimal levels of the proteins

44 involved. To achieve this, there is a network of factors that coordinate protein synthesis,

45 folding, localization, and degradation, known as the proteostasis network. Protein

46 degradation is mainly controlled by two pathways in eukaryotes, the Ubiquitin-

47 Proteasome System (UPS), and the autophagy-lysosome pathway (Finley et al., 2012;

48 Feng et al., 2014; Dikic, 2017). Substrates of the UPS are labeled with one or more

49 ubiquitins in a highly selective process (Hershko and Ciechanover, 1998; Schrader,

50 Harstad and Matouschek, 2009; Finley et al., 2012). In humans more than 600 ubiquitin

51 ligases are dedicated to the recognition and labeling of specific proteasome substrates

52 (Li et al., 2008). While many ubiquitinated proteins are recognized and degraded by

53 proteasomes, ubiquitination also serves other functions depending on the type of

54 ubiquitination and cellular state (Fujii et al., 2009; Shaid et al., 2013; Rittinger and Ikeda,

55 2017). The second pathway of protein degradation is the autophagy-lysosome system.

56 Here, macro-autophagy, hereafter referred to as autophagy, is the major pathway.

57 Autophagy (literally self-eating) involves the targeting of proteins to the concave side of

58 de novo formed double membrane structures. Upon expansion and closure of these

59 structures, an autophagosome is formed that has engulfed cytosolic cargo (Kabeya et al.,

60 2000; Feng et al., 2014; Dikic, 2017). Fusion of the outer membrane of these

61 autophagosomes with lysosome (vacuoles in yeast and plants) exposes the inner

62 membrane and engulfed material to acidic hydrolases that degrade the content.

63 Autophagy can be divided into selective and non-selective processes. These are

64 differentially regulated depending on the substrate and cellular conditions. While there is

65 overlap amongst proteasome and autophagy substrates, autophagy is uniquely capable

66 of clearing protein aggregates, damaged organelles, viruses, and large multi-subunit

67 complexes (Ogata et al., 2006; Kraft, Reggiori and Peter, 2009; Kamada et al., 2010;

68 Deffieu et al., 2013; Marshall et al., 2015; Mochida et al., 2015; Waite et al., 2015; BAO

69 et al., 2016).

$70 \quad$ Proteasomes are one of the large multi-subunit complexes that are degraded via

71 autophagy, a process referred to as proteaphagy. Proteaphagy appears to be a selective

72 process as it depends on factors not involved in general autophagy (Waite et al., 2015; 
73 Cohen-Kaplan et al., 2016; Marshall, McLoughlin and Vierstra, 2016; Nemec et al., 2017;

74 Li et al., 2019) and does not occur under several conditions that induce general autophagy

75 (Waite et al., 2021). A striking example of this is carbon starvation, which induces general

76 autophagy (Adachi, Koizumi and Ohsumi, 2017). Proteasomes, however, localize to

77 cytosolic punctate structures termed proteasome storage granules (PSGs) upon carbon

78 starvation (Laporte et al., 2008). While several factors that regulate PSG formation or

79 their subsequent dissolution have been identified (Peters Lee Zeev et al., 2013;

80 Weberruss et al., 2013; van Deventer et al., 2014; Marshall and Vierstra, 2018; Li et al.,

81 2019), the signals that trigger proteasome relocalization and the mechanisms that

82 regulate it have not been resolved.

83 Grown in the presence of glucose, yeast mainly utilize glycolysis for energy (ATP)

84 and biomass production, while suppressing mitochondrial respiration (Kayikci and

85 Nielsen, 2015). However, upon depletion of glucose, cells adapt and the metabolism

86 switches from mainly glycolysis to mitochondrial respiration. This process is facilitated by

87 autophagy, which provides a source of serine required for mitochondrial one-carbon

88 metabolism (May, Prescott and Ohsumi, 2020). While uniquely adapted to utilize glucose

89 as a carbon source, yeast grown in the presence of other carbon sources, like raffinose

90 or glycerol, primarily utilize mitochondrial respiration for energy production (Kayikci and

91 Nielsen, 2015; Adachi, Koizumi and Ohsumi, 2017). Interestingly, yeast do not induce

92 general autophagy when switched from glucose containing media to carbon starvation

93 media, presumably due to the lack of ATP. However, yeast grown on non-glucose carbon

94 sources prior to starvation do induce general autophagy upon removal of the carbon

95 source (Adachi, Koizumi and Ohsumi, 2017). This suggests the cellular respiratory state

96 and ATP levels are determinants in signaling autophagy. However, it should be noted that

97 the difference between glycolytic growth with repressed respiration and growth with active

98 mitochondrial respiration is also accompanied by a differences in the expression of

99 numerous genes (Galdieri et al., 2010).

100 As proteasomes and autophagy both contribute to the replenishment of cellular 101 metabolites, we hypothesized that the metabolic state of cells, like with autophagy, 102 impacts proteasomes as well. Here, we report the relocalization of proteasomes to PSGs 103 is restricted when yeast are starved following respiratory growth, but not glycolytic growth 
104 (where respiration is suppressed). Consistent with this, conditions that interfered with

105 mitochondrial function, such as inhibition of different electron transport chain complexes,

106 resulted in the formation of proteasome granules in yeast. This differential regulation

107 based on the initial carbon source involves specific signaling pathways: the AMP kinase

108 Snf1 and a MAP kinase signaling cascade, both regulate proteasome granule formation.

109 We propose a model where proteasome localization is regulated through mitochondrial

110 respiration.

Results

Proteasome Granule Formation is Restricted During Respiratory Growth.

The observation that the growth media prior to carbon starvation influenced yeast's

116 autophagic response resolved a controversy regarding general autophagy induction

117 following carbon starvation (Takeshige et al., 1992; Lang et al., 2014). Starvation

118 following respiratory growth (like in media containing raffinose or glycerol as the carbon

119 source), promoted autophagy induction while growth in glycolytic/fermenting media, like

120 dextrose (D-glucose), did not (Adachi, Koizumi and Ohsumi, 2017). Consistent with this,

121 a link between respiration and autophagy induction during energy deprivation has been

122 established (Yi et al., 2017).Thus, the metabolic state of the cell is an important variable

123 when evaluating carbon starvation responses. We previously showed that carbon

124 starvation does not induce proteasome autophagy in yeast (Waite et al., 2016), however

125 we did not specifically control for the pre-starvation condition of the cells. Therefore, we

126 used media containing dextrose (YPD, glycolysis), raffinose (YPR, glycolysis and

127 respiration), or glycerol (YPG, respiration exclusively) as sources of carbon to examine

128 proteaphagy following carbon starvation. We monitored proteasome autophagy with a

129 GFP-tag fused to the regulatory particle subunit Rpn1. Here, the observation of vacuolar

130 fluorescence in cells or the appearance of a faster migrating "free" GFP species on

131 immunoblots are indicative of proteaphagy. No robust proteaphagy was observed upon

132 switching to carbon starvation, independent of the growth medium prior to starvation. The 133 amount of "free" GFP detected at 1 and 2 days of carbon starvation was less than "free"

134 GFP detected following six hours of nitrogen starvation (Waite et al., 2015) (Fig. 1A). 
135 Instead, proteasome granules were induced, as previously reported (Waite et al., 2015;

136 Marshall and Vierstra, 2018). Interestingly, pre-growth in dextrose resulted in

137 approximately $58 \%$ of cells showing granules with $27 \%$ of these showing multiple

138 granules per cell, whereas pre-growth in raffinose resulted in less than $32 \%$ of cells

139 showing granules and $5 \%$ of these showing multiple granules per cell (Fig. 1B). A similar

140 trend was observed when we monitored the core particle using $\alpha 1$-GFP as a reporter

141 (S1). This suggest that proteasome localization is affected by the carbon source cells

142 were grown in prior to starvation.

143 To explore the role of nutrients in proteasome localization further, we analyzed the

144 induction of PSGs following prolonged growth in rich media, another condition that results

145 in PSG formation (Laporte et al., 2008; Peters et al., 2016). When granules formed after

146 growth for an extended period, a single dominant granule was observed in cells

147 regardless of the initial carbon source. However, we observed that cells grown in rich

148 media containing dextrose for 3 days had more cells forming granules in general than

149 cells grown in media containing raffinose. Growth in glycerol media resulted in very few

150 cells forming granules after 3 days (Fig. 1C). This was also observed with $\alpha 1-G F P$ as a

151 reporter (S2). Consistent with a model where PSGs protect proteasomes from autophagy

152 (Marshall and Vierstra, 2018), the conditions with less granules (i.e. raffinose and

153 glycerol) showed more "free" GFP on immunoblots at both 2 and 3 days of growth in

154 carbon containing media, (Fig. 1C, S3) suggesting an increase in proteaphagy. However,

155 we would expect more proteaphagy following growth in glycerol considering this condition

156 formed markedly fewer PSGs (Fig. 1C). Interestingly, we observed GFP-positive bands

157 on immunoblots with molecular weights between "free GFP" and Rpn1-GFP following

158 prolonged growth in glycerol. Such fragmentation patterns were recently observed

159 specifically for GFP-tagged proteasomes degraded through ESCRT-mediated micro-

160 autophagy (Li et al., 2019). In all, our data suggest that not only PSGs, but also the type

161 and magnitude of proteasome autophagy, is dependent on the initial carbon source and

162 the cell's metabolic state prior to starvation.

163 Switching from dextrose media to carbon starvation results in the formation of

164 multiple phagophore assembly sites (PAS), which does not occur when carbon starvation

165 is initiated following prior growth in other carbon sources or upon nitrogen starvation. This 
166 likely affects autophagy induction and might contribute to the lack of autophagy in this 167 condition (Adachi, Koizumi and Ohsumi, 2017). The number of proteasome granules 168 observed per cell correlates with increased PAS sites when comparing switching from 169 dextrose or raffinose to carbon starvation (Fig. 1B, S1). To determine if proteasomes co170 localized with PAS during carbon starvation, we used cells expressing GFP-Atg8 (PAS/ 171 autophagy marker) and Rpn1-mCherry. We monitored GFP and mCherry localization 172 upon carbon starvation after pre-growth in dextrose, raffinose, or glycerol containing 173 media (Fig. 1D). PAS structures were observed within 1 hour of starvation, when no PSGs 174 are yet detectable. At 24 hours when both PSG and PAS can be observed, we did not 175 observe any striking colocalization (Fig. 1D), indicating these are distinct structures. To 176 test if PSG formation depended on PAS formation or the signaling pathway that induces 177 an autophagic response upon carbon starvation, we analyzed strains deleted for different 178 genes required for autophagy induction: ATG1, SNF1 and GGC1. These gene products 179 activate Mec1 in a process that tethers Mec1 to the mitochondrial outer membrane upon 180 carbon starvation. This mitochondrial localization together with active mitochondrial 181 respiration are prerequisites for autophagy induction (Yi et al., 2017). ATG1, SNF1, and 182 GGC1 were not required for proteasome granule formation upon carbon starvation (S4) 183 indicating multiple distinct signals couple the cells metabolic state to these two 184 degradative machineries. The majority of cells formed PSGs at 24 hours when pre-grown 185 in dextrose ( $56 \%)$, which was significantly higher compared to cells pre-grown in 186 raffinose or glycerol (26\% and $22 \%$ respectively) (Fig. 1D). In sum, conditions where cells 187 initially had active respiration show less PSGs.

188 To further probe the role of mitochondrial respiration in regulating proteasome 189 localization, we tested other conditions that induce respiration vs glycolysis. First, we 190 tested the carbon starvation response after growing cells for 4 hours (more glycolysis and 191 fermentation) or 24 hours (more respiration) in YPD medium. After 24 hours of dextrose 192 depletion, yeast have switched to respiration for energy production (Galdieri et al., 2010). 193 Indeed, starving after 24 hours of growth in dextrose resulted in a reduction in granule 194 formation compared to 4 hours (S5). Next, we starved cells for phosphate following 195 growth in different carbon sources. This was based on the rationale that cells lacking 196 phosphate sources would be compromised in maintaining energy production as 
197 phosphate is required for ATP production (Ko, Hong and Pedersen, 1999). Phosphate 198 starvation induces cell cycle arrest and a stress response similar to carbon starvation 199 (Petti et al., 2011; Secco et al., 2012). Phosphate starvation also induced proteasome 200 autophagy but to a lesser extent than nitrogen starvation at 24 hours (Waite et al., 2021). 201 Here, we show that prolonged phosphate starvation induced proteasome granules 202 (Fig.1E). Like PSGs induced by carbon starvation, these granules had different properties 203 depending on the initial carbon source. Cells grown in raffinose that were starved for 204 phosphate showed less PSGs compared to cells grown in dextrose. Even more striking 205 was the almost complete absence of granules when cells were grown in glycerol (Fig. 206 1E), even though our phosphate starvation media always contained dextrose as a carbon 207 source. Catabolite repression does not appear to play a role here as raffinose and glycerol 208 pre-growth resulted in distinct phenotypes. Because granules induced by phosphate 209 starvation are evident only at later timepoints (2 days), they could result from prolonged 210 growth similar to granules formed during stationary phase. However, we found granules 211 were induced to a much larger extent in SD media lacking phosphate compared to SD 212 complete media, both of which contained dextrose as a carbon source (S6). In all, 213 proteasome granule formation appears to be in part dependent on mitochondrial 214 respiration. Under conditions of limited mitochondrial respiration, proteasomes appear to 215 re-localize to PSGs more efficiently.

\section{Mitochondrial Inhibition Induces Proteasome Granule Formation}

218 Cells grown in respiratory media formed fewer PSGs when starved for carbon, 219 phosphate, or grown for three days. Thus, increased mitochondrial function appears to 220 limit PSG formation. This led to the hypothesis that reducing mitochondrial function has 221 opposing effect and causes proteasome granule formation. To test this, we inhibited 222 mitochondrial function by targeting different components of the electron transport chain 223 (ETC). Sodium azide, antimycin A, and oligomycin A were used to target complex IV, 224 complex III, and ATP synthase respectively. We further utilized the uncoupler CCCP to 225 disrupt ATP synthesis (Heytler and Prichard, 1962; Wikström and Berden, 1972; Gribble 226 et al., 1997; Symersky et al., 2012). All four drugs induced proteasome granules in rich 227 (dextrose) media (Fig. 2A), further corroborating the link between mitochondrial function 
228 and proteasome localization. To test if granules induced through mitochondrial inhibition

229 behave like proteasome granules induced by carbon starvation, we tested the reversibility

230 of these granules. Carbon starvation induced PSGs, unlike other types of proteasome

231 containing granules, quickly disappear with proteasomes re-localizing to cell nuclei upon

232 re-addition of a carbon source (Laporte et al., 2008; Peters Lee Zeev et al., 2013;

233 Weberruss et al., 2013; Waite, Burris and Roelofs, 2020). Granules induced by

234 mitochondria inhibitors quickly dissipated and GFP signal was predominantly nuclear

235 after cells were washed and re-inoculated into drug free media (Fig. 2B, S7). This

236 indicates that these granules are not associated with irreversible aggregates but are

237 dynamic structures consistent with the proposed storage function of PSGs. Similar to ETC

238 inhibitors, we predicted the absence of the final electron acceptor, oxygen, should also

239 result in PSG formation by inhibiting oxidative phosphorylation. Indeed, upon growth in

240 anoxic conditions, proteasome granules were induced (S8). Like with carbon starvation,

241 the magnitude of mitochondria inhibition-induced granules was dependent on the carbon

242 source during initial growth with the exception of Rpn1-GFP cells treated with antimycin

243 A (Fig. 2C). These data further indicate a role for mitochondrial respiration in regulating

244 proteasome granule formation.

245 Intriguingly, we did not detect induction of proteasome granules with all

246 mitochondria inhibitors tested. Surprisingly, potassium cyanide (KCN) treatment, which

247 like azide targets cytochrome c oxidase, did not result in any significant granule formation

248 at various concentrations (Fig. 2D). The KCN treated cells grew slower than untreated

249 cells (with a growth rate comparable to other mitochondria inhibitors) and we observed

250 an increase in cell size at high concentrations (Fig. 2D), indicating KCN treatment was

251 effective. Apparently mitochondrial inhibition or stress alone is not sufficient for PSG

252 formation.

253 Mitochondria play a key role in efficient ATP production and ATP is essential for 254 proteasome function (Finley, 2009; Schrader, Harstad and Matouschek, 2009).

255 Furthermore, proteasome complexes are unstable in vitro in the absence of ATP (Kleijnen

256 et al., 2007). Therefore we wondered if ATP maintenance and proteasome stability are

257 key determinants in PSG formation; something that has been postulated before (Enenkel,

258 2018; Karmon and Ben Aroya, 2020). To test if PSG formation was linked to ATP 
259 production, we measured ATP under PSG inducing and non-inducing stimuli. To do this, 260 we utilized the Cell Titer Glo as well as the Enliten ATP assay systems (Nicastro et al., 261 2015; Adachi, Koizumi and Ohsumi, 2017). Inhibiting mitochondria with sodium azide, 262 antimycin A, oligomycin A, CCCP and growing yeast anoxically, led to a large reduction 263 in ATP compared to growth in rich media (Fig. 2E). This reduction in ATP levels correlated 264 with PSG formation (Fig. 2E). Interestingly, KCN treatment caused a smaller reduction in 265 ATP levels compared to the untreated control, and this inhibitor did not induce PSGs. We next measured ATP under starvation conditions. We observed a larger reduction in ATP following nitrogen starvation than carbon starvation, while only carbon starvation induced granule formation. Similarly, we observed a strong reduction in ATP detected following 24 hours of phosphate starvation, a condition that induces proteasome autophagy with PSGs observed 48 hours post starvation (Fig. 1F) (Waite et al., 2021).Thus, with starvation, a reduction in ATP levels by itself does not trigger proteasomes re-localization to PSGs. One possible explanation is that in addition to a drop in ATP levels, other signals are generated during nitrogen and phosphate starvation that result in proteaphagy.

\section{MAP Kinase Signaling is Required for Proteasome Granule Formation}

The differential regulation of proteasome granules observed following respiratory vs non-respiratory growth, as well as the observation that not all mitochondria inhibitors induce proteasome granules (Fig. 2D), suggests that there is some regulator of this process that is only active under specific conditions. The cell wall integrity MAP kinase cascade proteins Mpk1(Slt2), Mkk1, and Mkk2 are known to regulate proteasome abundance and proteasome autophagy (Rousseau and Bertolotti, 2016; Waite et al., 2021). This kinase cascade has further been shown to be required for induction of general autophagy when antimycin A or potassium cyanide were added to cells in a process that was dependent on Atg32 and Atg11 (Deffieu et al., 2013). When we tested if deletion of ATG32 disrupted the formation of proteasome granules induced after prolonged yeast growth, azide or antimycin A treatment, we observed no reduction in PSG formation (S9). Similarly, ATG11 was not required for granule formation when cells were treated with sodium azide (S10). This shows that the requirements for autophagy induction and PSG 
290 formation are not identical in these conditions. Next, we wanted to test the role of MAP 291 kinase signaling itself. To test if the MAPK Mpk1 was important for PSG formation, we 292 deleted the MPK1 gene. In these cells we observed a striking difference depending on

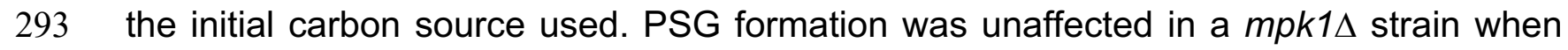
294 starved for carbon following initial growth in dextrose (Fig. 3A, E). However, when yeast 295 were grown in raffinose prior to starvation, Mpk1 was critical to form granules efficiently 296 for both the RP (Rpn1) and CP ( $\alpha 1)$ reporters (Fig. 3A, E). This suggest that Mpk1 was 297 required for proteasome granule formation in cells that had active mitochondrial 298 respiration. When we monitored granule formation following oligomycin $A$, antimycin $A$ 299 and CCCP treatment, we found a similar requirement for Mpk1 when cells were grown in 300 raffinose (Fig. 3B-D, F). With respect to sodium azide, granules monitored using a GFP301 tag on the CP subunit $\alpha 1$ behaved as above with a reduction in the MPK1 mutant following growth in raffinose (Fig. 3E, F). Granule formation monitored using GFP-tag on the RP 303 subunit Rpn1, however, was restricted when mpk1 cells were grown in dextrose but 304 not raffinose (Fig. 3E, F). This observation further supports the idea of differential 305 regulation of $\mathrm{CP}$ and $\mathrm{RP}$ in granule formation, as has been observed before (Weberruss 306 et al., 2013; Marshall and Vierstra, 2018; Karmon and Ben Aroya, 2020). Next, we tested 307 for a role of the proteasome chaperone Adc17. Adc17 is activated under several stress 308 conditions (Hanssum et al., 2014) by Mpk1 (Rousseau and Bertolotti, 2016). However, 309 Adc17 was dispensable for PSG formation, suggesting an alternate route of proteasome 310 regulation by Mpk1(S11).

311 To determine the involvement of other kinases, we analyzed a number of proteins 312 within the cell integrity pathway, as well as other major yeast MAPKs from independent 313 pathways (Levin, 2005). The deletion of FUS3 or HOG1 (MAPKs involved in pheromone 314 response and osmoregulation respectively) did not prevent or substantially reduce 315 proteasome granule formation (S11). This indicates a specific role for the cell integrity 316 kinase pathway. Looking at MAP kinase kinases upstream of Mpk1, we evaluated Mkk1 317 and Mkk2. While individual deletion of these paralogs had little impact on granule 318 formation, a double deletion of $M K K 1$ and $M K K 2$ showed only few proteasome granules 319 under carbon starvation, azide treatment, antimycin A, oligomycin A and CCCP treatment 320 (Fig. 3F). This reduction was, like with Mpk1, observed when cells were grown in raffinose 
321 but not glucose prior to treatment. Tracing this MAP kinase cascade further, we found

322 that the MAPKKK Bck1 was similarly required, however these cells formed more granules

323 after switching from glucose media than MPK1 or MKK1/MKK2 mutants. Though we did

324 observe a more significant reduction compared to wildtype when cells were cultured in

325 raffinose (Fig. 3G). Upstream of Bck1, the transmembrane activator of the cascade,

326 Wsc1, was also required for granule formation (S12). Other upstream activators of the

327 cell integrity pathway, namely Wsc2, Wsc3 and Ack1 (Verna et al., 1997; Kuranda et al.,

328 2006; Krause, Xu and Gray, 2008), were not required for PSG formation under the same

329 conditions (S12). Our data show that an intact map kinase pathway, starting at Wsc1 and

330 moving downstream to Mpk1, is required for proteasome granule formation when cells

331 are grown in respiration inducing conditions. We have previously reported that Mpk1,

332 Mkk1 and Mkk2 are required for efficient proteaphagy (Waite et al, 2021). Both conditions

333 require nuclear export of proteasomes, potentially indicating this kinase cascade

334 regulates nuclear export of proteasomes, for example by Mpk1 directly phosphorylating

335 proteasomes. However, Mpk1 is involved in many cellular processes and this kinase

336 pathway might be indirectly involved in signaling proteasome re-localization. These

337 possibilities are currently being tested.

Snf1 is required for proteasome granule formation upon mitochondrial inhibition

As mentioned above, Snf1 is required for the induction of autophagy upon glucose starvation. This kinase is recruited to mitochondria shortly after the onset of glucose

342 starvation where it phosphorylates Mec1. Phosphorylated Mec1 recruits Atg1 to

343 mitochondria and both factors are required for maintaining mitochondrial respiration

344 during glucose starvation (Yi et al., 2017). This respiration in turn is also required for

345 autophagy induction (Adachi, Koizumi and Ohsumi, 2017; Yi et al., 2017). We did not

346 observe a reduction in PSGs upon carbon starvation when SNF1 or other factors involved

347 in this pathway were deleted (S4), a finding consistent with published data (Li et al., 2019).

348 Snf1 was also not required for proteasome autophagy induced by nitrogen starvation, but

349 was required for proteasome autophagy observed following 4 days of growth in SC media 350 with $0.025 \%$ (low) glucose (Li et al., 2019). Intriguingly, we noticed a different role for Snf1 351 in proteasome autophagy under growth with different carbon sources. Deletion of SNF1 
352 promoted autophagy of proteasomes when cells were grown for 3 days in rich media 353 containing dextrose or glycerol but not raffinose (Fig. 4A). Further, we observed that 354 SNF1 mutants failed to form proteasome granules efficiently under these conditions (Fig. 4B). This is consistent with a model whereby PSGs protect proteasome from autophagic 356 degradation (Marshall and Vierstra, 2018), however, we did not observe increased 357 autophagy when cells were grown in raffinose, despite the failure in granule formation in 358 this condition. Here, GFP signal appeared more nuclear and diffuse in the cytoplasm for 359 the Snf1 mutant than the wildtype which formed PSGs (Fig. 4B). The disparate 360 phenotypes we observed likely reflect the complexity of combined inputs to cells 361 metabolic state and mitochondrial respiration activity in regulating proteasome 362 localization.

To gain more insight into the role of Snf1 in regulating proteasomes localization, 364 autophagy, and granule formation, we tested the effect of mitochondria inhibitors in these 365 cells. Unlike granules induced by carbon starvation, granules induced with mitochondria 366 inhibitors were highly dependent of Snf1 (Fig. 4C) for both the RP and CP tag reporters 367 (Li et al., 2019). Upon mitochondrial inhibition, compared to wild type, Snf1 mutants have 368 fewer proteasome granules, more nuclear GFP signal and little to no vacuolar GFP signal. 369 Of note, these cells were grown in glucose where Snf1 is inactive (Schüller, 2003; Kayikci 370 and Nielsen, 2015). This may indicate an alternative role for Snf1 in regulating 371 proteasome localization outside of its canonical role of non-fermentative gene repression. 372 In all, these data suggest that different pathways are involved in the formation of 373 proteasome storage granules, for example during carbon starvation versus mitochondria 374 inhibition. This raises the question to what extent these granules are similar or 375 qualitatively different in function or regulation.

\section{Discussion}

The transcriptional upregulation of proteasomes (by Rpn4 in yeast and Nrf1 in mammals) is critical for cells to respond to various stress conditions. However, not all 380 stresses require a (continuous) upregulation of proteasomes and instead trigger either 381 the degradation of proteasomes or their relocalization into cytosolic granules. In yeast, 382 proteasome complexes are enriched in the nucleus under optimal conditions such as 
383 during logarithmic growth. However, proteasomes re-localize to cytoplasmic granules 384 upon carbon limitation (Enenkel, 2012; Peters Lee Zeev et al., 2013; Waite et al., 2015; 385 Marshall and Vierstra, 2018). When stressed for nitrogen, on the other hand, proteaphagy 386 is induced (Marshall et al., 2015; Waite et al., 2015; Nemec et al., 2017). Similarly, in 387 human cells, proteasomes have been reported to undergo proteaphagy in response to 388 amino acid starvation or upon proteasome inhibition (Cohen-Kaplan et al., 2016; Choi et 389 al., 2020; Goebel et al., 2020). Interestingly, cytoplasmic proteasomes appear to be 390 substrates for proteaphagy upon amino acid deprivation while nuclear proteasomes 391 undergo liquid-liquid phase separation (LLPS) (Uriarte et al., 2021). Osmotic stress has 392 also been shown to induce nuclear LLPS granules of proteasomes (Yasuda et al., 2020). 393 Thus, both in yeast and humans depending on the condition, proteasomes show distinct 394 fates and localization. To understand how these specific fates of proteasomes are 395 regulated, we need to not only know the factors involved, but also understand the triggers 396 responsible. This can be rather subtle, as it was recently shown that clearly defined fates 397 for proteasomes were observed when comparing cells starved with low levels of glucose 398 or no glucose at all ( $\mathrm{Li}$ et al., 2019). Here, only the cells starved in low glucose induced 399 Snf1-dependent micro-autophagy of proteasomes. In the current study, we sought to 400 determine in detail what triggers proteasome granular localization and further identified some of the key signaling kinases involved.

\section{Mitochondrial Respiration as key determinant.}

Our data show that mitochondrial respiration, at least in part, regulates proteasome 405 localization (Figs. 2, 5). First, we show that several inhibitors of mitochondrial respiration 406 robustly induce PSG formation. Second, removing a carbon source from cells that grew 407 in media that suppresses respiration (i.e. with glucose (Galdieri et al., 2010)) resulted in 408 the formation of multiple granules per cell, whereas one dominant granule was present 409 when cells were switched from media that required respiration (glycerol or raffinose). This 410 observation shows interesting parallels with the regulation of general autophagy upon 411 carbon starvation that was recently shown to be dependent on respiration (Adachi, 412 Koizumi and Ohsumi, 2017). Another study found a complex of Snf1-Mec1-Atg1 is 413 recruited to the mitochondrial membrane by Ggc1 following the onset of carbon 
414 starvation. The formation of this complex is required to maintain active mitochondrial 415 respiration which in turn is required to initiate carbon starvation induced autophagy (Yi et 416 al., 2017). Surprisingly, the deletion of GGC1 did not impact PSG formation, suggesting 417 additional signaling pathways link respiration to the regulation of proteasome localization. 418 The extent of granule formation when cells were grown in different carbon sources 419 negatively correlated with the amount of general autophagy induced, as we detected a 420 reduction in the number of PSGs under conditions that led to increased general 421 autophagy upon carbon starvation (Adachi, Koizumi and Ohsumi, 2017). Here, we 422 observed only a small increase in proteaphagy (Fig. 1A) when cells were grown in 423 respiratory media, suggesting the reduced levels of PSGs cannot be explained by 424 increased autophagy of proteasomes. This is in contrast to the reported role of PSGs in 425 protecting proteasomes from autophagy as we would expect more proteasome 426 autophagy when there is a reduction in PSGs. Our data suggest that proteasomes are 427 excluded from autophagic degradation without being sequestered into PSGs. This may 428 indicate a role for proteasome activity in regulating the autophagic response of respiring 429 cells. Further supporting our observations is the lack of an increase in proteasome 430 autophagy under autophagic conditions in mutants that are defective in granule formation 431 (Figs. 3 and 4). The nuclear enrichment of fluorescence we observed under these 432 conditions indicates that the majority of proteasomes remained nuclear. This localization 433 shields proteasomes from general autophagy (Waite et al., 2015; Nemec et al., 2017). 434 Thus, proteasomes can be excluded from autophagic degradation either by PSG 435 formation or by nuclear retention. In line with this, the deletion of autophagy related genes 436 results in nuclear retention (Waite et al., 2015, 2021) This suggest proteasome 437 relocalization is tightly regulated and below we discuss a kinase cascade we have 438 identified that is involved in this process.

\section{Kinases that regulate proteasome localization.}

441 MAP kinases and Snf1 signaling pathways both regulate proteasome localization, 442 however, each under distinct conditions. The Mpk1 kinase cascade was required 443 following growth in media that induced respiration, while Snf1 was required upon 444 mitochondrial inhibition (but not carbon starvation). These pathways are known to 
445 cooperate upon cell wall and oxidative stress (Backhaus et al., 2013; Willis et al., 2018), 446 indicating they might work together to regulate proteasomes more generally. Indeed, both 447 pathways are also involved in regulating proteasome abundance and mobilization upon 448 different stressors. Mpk1 is required for proteasome upregulation upon ER stress 449 (Rousseau and Bertolotti, 2016; Schmidt et al., 2019) as well as required for efficient 450 proteaphagy (Waite et al., 2021), and Snf1 is required for micro-autophagy of 451 proteasomes following growth in low glucose media (Li et al., 2019). The requirement for 452 these pathways in both proteasome autophagy and proteasome granule formation could 453 indicate they are necessary for efficient nuclear export of proteasomes, a pre-requisite 454 for both autophagy and granule formation (Nemec et al., 2017). However, the possibility 455 that they play a less direct role in regulating proteasome localization cannot be excluded. 456 It has been shown that MPK1 is required for general autophagy induction when 457 mitochondria are inhibited with the drug antimycin A (Deffieu et al., 2013). Similarly, it is 458 required for mitophagy and pexophagy induced following nitrogen starvation, even though 459 general autophagy does not depend on Mpk1 (Mao et al., 2011). Mitophagy here was 460 shown to depend on the complete the cell integrity MAPK cascade (from the 461 transmembrane receptor kinase Wsc1 to Mpk1). We demonstrate that this same cascade 462 is required for proteasome granule formation. These data point to a more general 463 signaling role for MPK1, particularly when mitochondria are affected. However, other 464 factors that are required for mitophagy under this conditions, such as Atg32, and Atg11, 465 had no effect on the formation of proteasome granules, suggesting that a general failure 466 in mitophagy does not correlate with a failure in proteasome granule formation. 467 Nevertheless, the MPK1 kinase cascade is clearly important in regulating proteasome 468 localization, particularly following respiratory growth.

469 An intact MAP kinase cascade is not sufficient to promote proteasome granule 470 formation. We report here that deletion of SNF1 restricted proteasome granule formation 471 upon inhibition of mitochondria. It was previously shown that this kinase is not required 472 for granule formation when cells were starved for glucose (Li et al., 2019). The 473 requirement for this protein in these different context suggests that proteasome granules 474 induced upon carbon starvation versus mitochondrial inhibition are distinct. It is intriguing 475 that Snf1 was required for granule formation when cells were grown in glucose rich media, 
476 as this protein is known to be inactive in this condition (Schüller, 2003; Turcotte et al., 477 2010). This suggests an unidentified role for Snf1 in this context that regulates 478 proteasome localization.

479 In all our data show that proteasomes are specifically regulated under different 480 metabolic conditions. This may indicate that proteasome activity is similarly regulated 481 based on cellular needs. Given that the majority of proteasomes are nuclear, proteasome 482 capacity and activity must be altered when they re-localize. Indeed, the current literature 483 suggests that proteasomes are inactive in PSGs (Gu et al., 2017; Enenkel, 2018). 484 Although it should be noted that LLPS structures induced by osmotic stress in HCT116 485 colon cancer cell line appear to be actively degrading ribosomal proteins (Yasuda et al., 486 2020). Under conditions of mitochondrial respiration where proteasomes are more 487 nuclear, proteasome activity may be necessary. This observed relationship between 488 mitochondrial respiration and proteasome localization is intriguing, as other studies have 489 demonstrated a functional link between mitochondria and proteasomes (Bragoszewski et 490 al., 2013; Bragoszewski, Turek and Chacinska, 2017; Lavie et al., 2018). This is also 491 evident in Parkinson's disease where proteasomes play a crucial role in regulating 492 mitochondrial dynamics (Junn et al., 2002; Ciechanover and Brundin, 2003; Webb et al., 493 2003; Um et al., 2010). Further, proteasomes are required to resolve mitochondrial stress 494 induced upon transporter clogging or accumulation of misfolded mitochondrial proteins in 495 the cytoplasm (Boos et al., 2019). Our data show that proteasomes are responsive to 496 cellular metabolism and are regulated differently depending on the cell's metabolic status. 497 While ATP appears to not be the trigger for PSG formation, at least upon starvation, our 498 data make it clear that mitochondrial function and signaling play an important role. 


\section{Materials and Methods}

\section{Yeast Strains}

504 All strains used in this study are reported in table S1. Our background strains are the 505 W303 derived SUB61 (Mata, lys2-801 leu2-3, 2-112 ura3-52 his3- $\Delta 200$ trp1-1) that arose 506 from a dissection of DF5 (Finley, Özkaynak and Varshavsky, 1987). Standard PCR based 507 procedures (primers and plasmids presented in table S2) were used to delete specific 508 genes from the genome, or introduce sequences at the endogenous locus that resulted 509 in the expression of C-terminal fusions of GFP or mCherry (Goldstein and McCusker, 510 1999; Hailey, Davis and Muller, 2002; Janke et al., 2004).

\section{Yeast Growth Conditions}

513 Overnight cultures of yeast were inoculated at an $\mathrm{OD}_{600}$ of 0.5 and grown in yeast extract 514 peptone (YEP) medium supplemented with $2 \%$ dextrose, raffinose or glycerol as a carbon 515 source and grown to an OD 6001.5 (approximately 4 hours). To induce starvation, cultures 516 growing logarithmically were centrifuged, washed with respective starvation medium, re517 inoculated at an $\mathrm{OD}_{600}$ of 1.5 , and incubated at $30{ }^{\circ} \mathrm{C}$ with constant shaking. Yeast 518 nitrogen base lacking carbon or phosphate sources was used to make the respective 519 starvation media. For drug treatments, cultures were grown to an $\mathrm{OD}_{600}$ of 1.5 as above, 520 then treated. Sodium azide (VWR), antimycin A (Sigma), oligomycin A (Cayman 521 Chemical) and 2-[2-(3-chlorophenyl)hydrazinylidene]-propanedinitrile (CCCP) (Sigma), 522 were used at final concentrations of $0.5 \mu \mathrm{M}, 0.1 \mathrm{mM}, 2.5 \mu \mathrm{M}$ and $10 \mu \mathrm{M}$ respectively. 523 Potassium cyanide (Fisher) was used at concentrations shown in Fig. 2E. Anoxic growth 524 was performed by transferring $1.8 \mathrm{~mL}$ of logarithmically growing culture to a $2 \mathrm{~mL}$ culture 525 tube that was sealed and incubated without shaking for 24 hours at $30{ }^{\circ} \mathrm{C}$.

\section{Protein Lysates and Electrophoresis}

528 For western blots, 2 ODs of cells were collected at indicated timepoints and treatments 529 and stored at $-80{ }^{\circ} \mathrm{C}$. Lysis was completed using previously establish methods 530 (Kushnirov, 2000). Following electrophoreses, samples were transferred to PVDF 531 membranes and immuno-blotted for GFP then Pgk1 followed by the appropriate 532 horseradish-peroxidase conjugated secondary antibodies. Antibodies used were anti- 
533 GFP (1:500; Roche, \#11814460001), and anti-Pgk1 (1:10,000; Invitrogen, \#459250).

534 Horseradish-peroxidase activity was visualized using the Immobilon Forte Western HRP

535 substrate (Millipore), and images were acquired using the G-box imaging system

536 (Syngene) with GeneSnap software. Genetools was used to quantify the amount of free

537 GFP normalized to the Pgk1 loading control.

\section{ATP Measurements}

540 Enliten ATP assay: 1 OD of cells was collected following indicated treatments and frozen 541 in liquid nitrogen. Pellets were resuspended in $50 \mu \mathrm{L}$ of $2.5 \%$ trichloro-acetic-acid (TCA) 542 and boiled for 3 mins. Sample was centrifuged (13,000 RPM) for 1 min and $2 \mu \mathrm{L}$ 543 supernatant was added to $98 \mu \mathrm{L}$ of $25 \mathrm{mM}$ Tris-HCL [pH 8.8] (1:50 dilution). $10 \mu \mathrm{L}$ of 1:50 544 dilution was combined with $40 \mu \mathrm{L}$ of $25 \mathrm{mM}$ Tris-HCL [pH 8.8] in a white 96 well plate. 50 $545 \mu \mathrm{L}$ of rLuciferase/Luciferin reagent (Promega, ENLITEN® ATP Assay System) was added 546 to each well and luminescence was monitored using a plate reader.

547 Cell Titer Glo ATP assay: 0.5 OD of cells were centrifuged following indicated 548 treatments and resuspended in $50 \mu \mathrm{L}$ of sterile water. Samples were transferred to a 96 549 well black plate and $50 \mu \mathrm{L}$ of Cell Titer Glo $2.0 \circledR$ reagent was added. This plate was 550 incubated in the dark under constant rotation on an orbital shaker for 4 minutes. Following 551 rotation, the plate was further incubated in the dark for 10 minutes at room temperature. 552 Luminescence was then measured using a plate reader.

554 Fluorescence Microscopy

555 All microscopy was performed with live yeast where proteasome subunits Rpn1 or $\alpha 1$ 556 was C-terminally tagged at their endogenous locus with expression driven by the 557 endogenous promoter. GFP-Atg8 was produced as previously described (Li et al., 2015). 558 After indicated treatments, approximately 2 ODs of cells were pelleted, washed with PBS, 559 then resuspend in $30 \mu \mathrm{L}$ of PBS. $3 \mu \mathrm{L}$ of this sample was then mounted on $1 \%$ soft agar 560 slides as described by E. Muller (https://www.youtube.com/watch?v=ZrZVbFg9NE8) 561 (Sundin et al., 2004). All imaging by fluorescence microscopy was done within 10 mins 562 following wash to avoid the effects of prolonged incubation on slides. Images were 563 acquired at room temperature using a Nikon Eclipse TE2000-S microscope at 600X 
564 magnification with a Plan Apo 60x/1.40 objective equipped with a Retiga R3 ${ }^{\text {tm }}$ camera

565 (Qlmaging). Images were collected using Metamorph software (Molecular Devices) and analyzed using FIJI. All quantification was performed using FIJI.

\section{Acknowledgements}

We thank Dr. Stella Lee for helpful discussions and feedback on the manuscript. We thank Mandeep Kaur for the generation of some of the yeast strains used in this study and feedback on the manuscript. We thank Dr. Alicia Burris for sharing her observation of proteasome granule formation under hypoxic conditions.

\section{Competing interests}

575 The authors declare no competing or financial interests.

\section{Author contributions}

578 Conceptualization: K.A.W., J.R.; Methodology: K.A.W., J.R.; Validation: K.A.W., J.R.; 579 Formal analysis: K.A.W., J.R.; Investigation: K.A.W.; Writing - original draft: K.A.W.;

580 Writing - review \& editing: K.A.W., J.R.; Visualization: K.A.W., J.R.; Funding acquisition: 581 J.R.

\section{Funding}

584 This work was supported by grants from the National Institutes of Health and National Institute of General Medical Science (K-INBRE program P20GM103418 and R01GM118660 to J. R.). The content is solely the responsibility of the authors and does not necessarily represent the official views of the National Institutes of Health.

\section{Supplementary information}

590 Supplementary information contains tables S1 and S2, supplementary figures S1-S12 591 and complete images of immunoblots used. 
bioRxiv preprint doi: https://doi.org/10.1101/2022.01.13.476202; this version posted January 13,2022 . The copyright holder for this preprint (which was not certified by peer review) is the author/funder, who has granted bioRxiv a license to display the preprint in perpetuity. It is made available under aCC-BY-NC 4.0 International license.

\section{Figures}

A

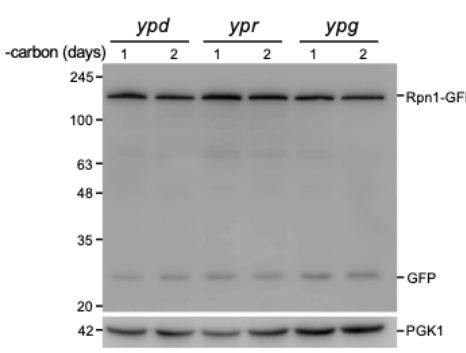

C

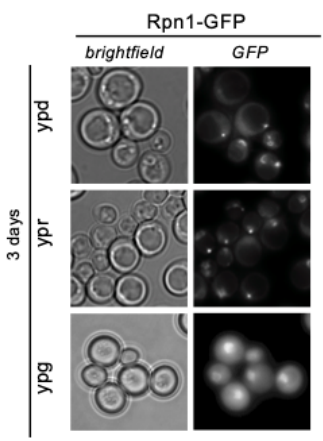

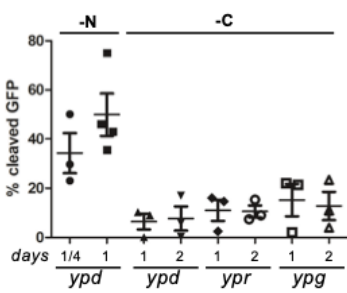
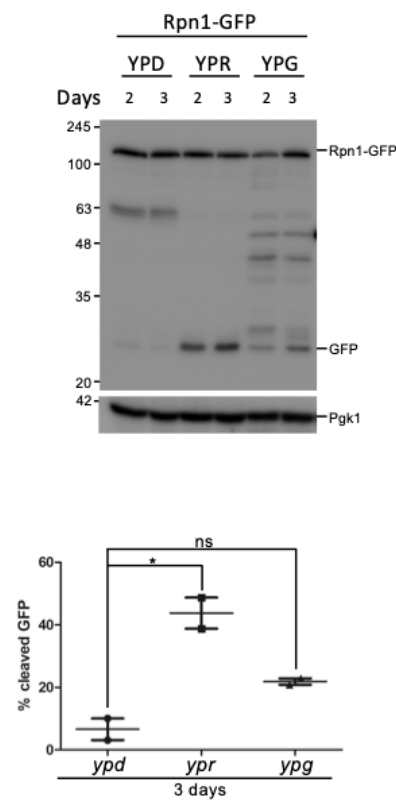

E

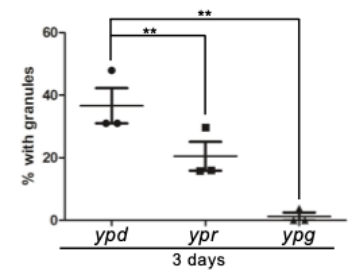

Eays
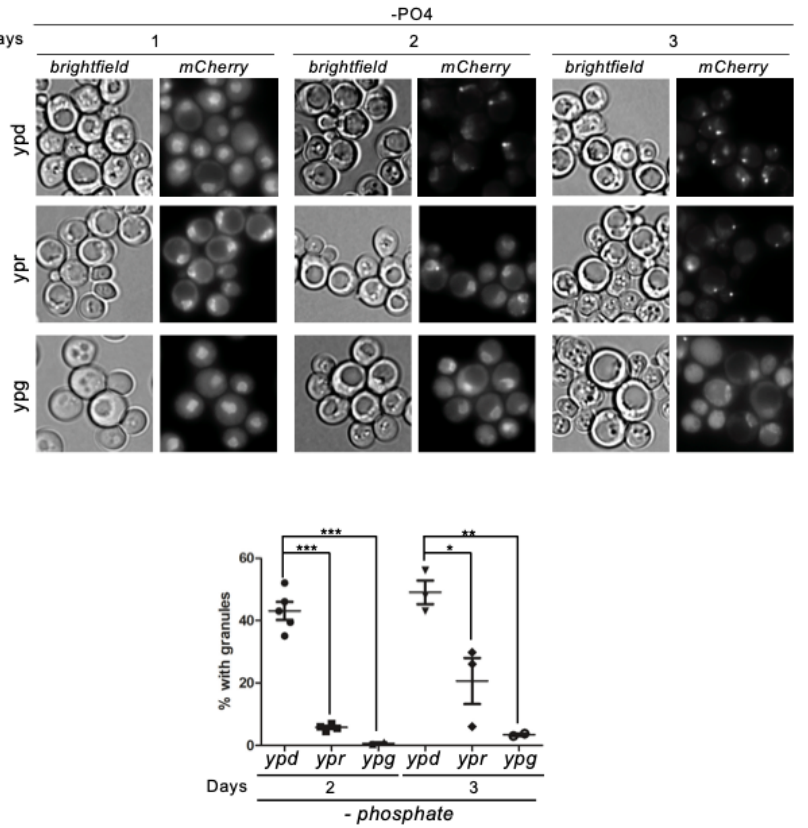

B

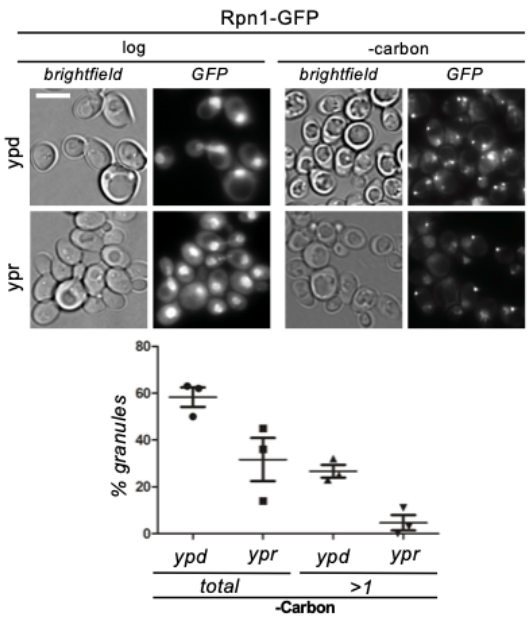

D
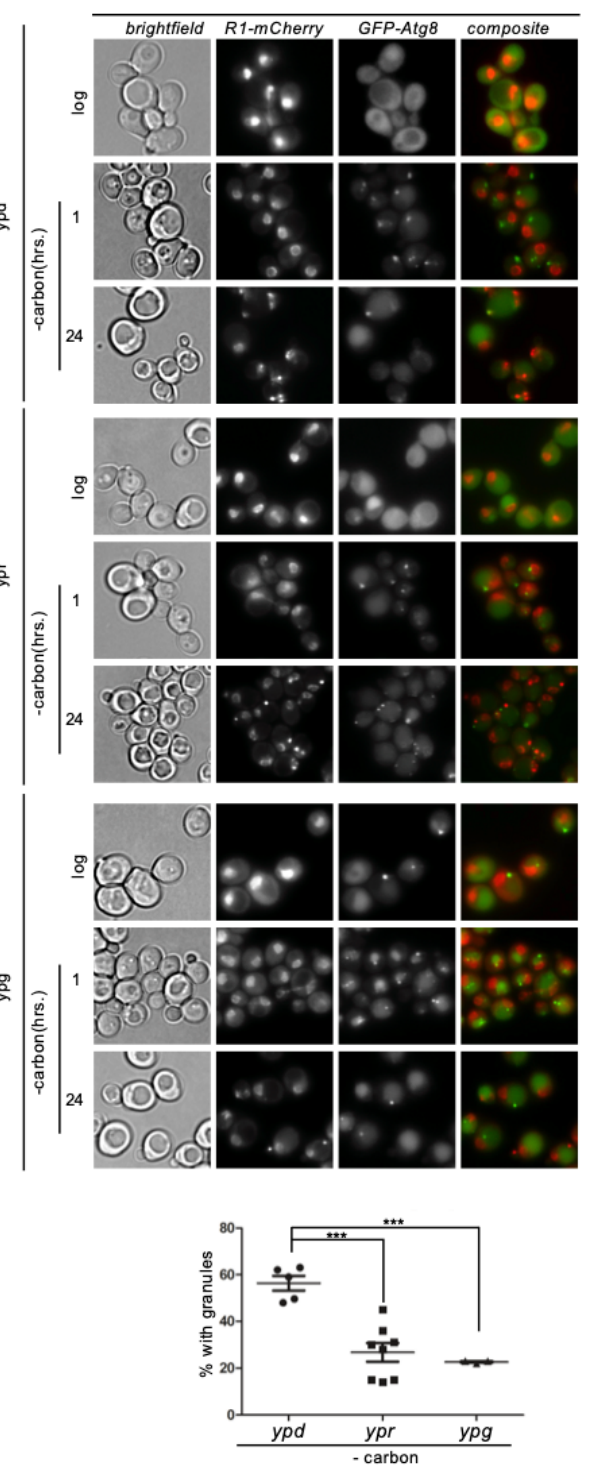
Figure 1. Proteasome granule formation is restricted during respiratory growth. (A) Rpn1-GFP expressing cells were grown for four hours in rich media containing dextrose (ypd), raffinose (ypr) or glycerol (ypg), followed by growth in SD media lacking carbon. 2 ODs of cells (i.e., the cells equivalent to two $\mathrm{ml}$ of culture $\mathrm{OD}_{600} 1$ ) were

600 harvested at indicated times and lysed as described in materials and methods. Lysates were separated by SDS-PAGE

601 and immunoblotted for GFP and Pgk1. Data presented are representative of three independent experiments.

602 Quantifications show the percentage of free GFP relative to the total amount of GFP (i.e., unprocessed + free GFP)

603 observed following nitrogen or carbon starvation. (B) Cells grown as in A were imaged at log phase and following 24

604 hours carbon starvation. Quantification shows the percentage of cells that induced proteasome granules and the percent

605 of those cells that formed two or more granules (Data presented are representative of three independent experiments

606 with $\mathrm{n}>100$ for each). Scale bar represents $5 \mu \mathrm{m}$. (C) (left) Rpn1-GFP expressing cells were grown for 3 days in the

607 indicated media then imaged microscopically. Quantification shows the percent of cells with granules from three

608 independent experiments. Statistical significance was determined with paired t-test (left) and $n>100$ for each datapoint.

609 (right) 2 ODs of cells were collected from cultures as in the left panel and lysed for immune blotting against GFP or

610 Pgk1. Data presented are representative of two independent experiments. Quantification shows the percentage of free

611 GFP, which indicating proteasome autophagy, relative to total GFP. Significance was determined using an unpaired

612 t-test. (D) Rpn1-mCherry (R1-mCherry); GFP-Atg8 expressing cells were grown in rich dextrose (ypd), raffinose

613 (ypr) or glycerol (ypg) media followed by a change to carbon starvation media. Microscopy was performed at indicated

614 times and quantifications show the percentage of cells with granules after 24 hours. Unpaired t-test was used to

615 determine significance and $\mathrm{n}>100$ for each data point. (E) Rpn1-mCherry expressing yeast were grown in indicated

616 rich media for 4 hours, washed and switched to phosphate starvation media. Microscopy was performed at the

617 indicated times. Quantification shows the percent of cells that formed granules at 2 and 3 days of phosphate starvation.

618 Unpaired t-test was used to determine significance with $\mathrm{n}>100$ for each datapoint. 
A

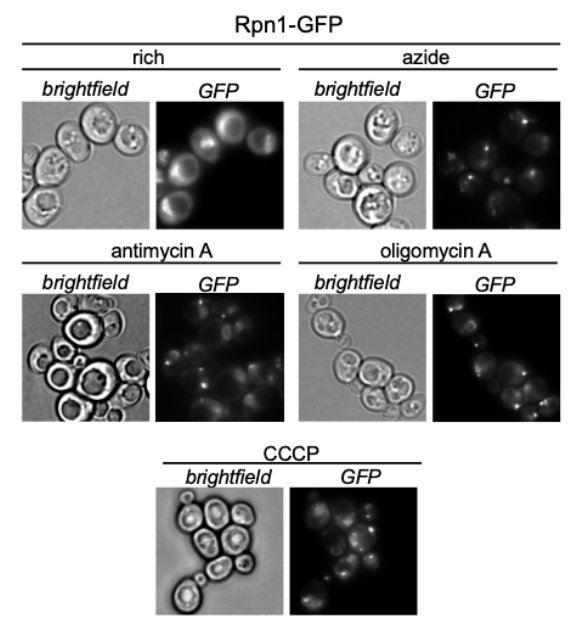

B

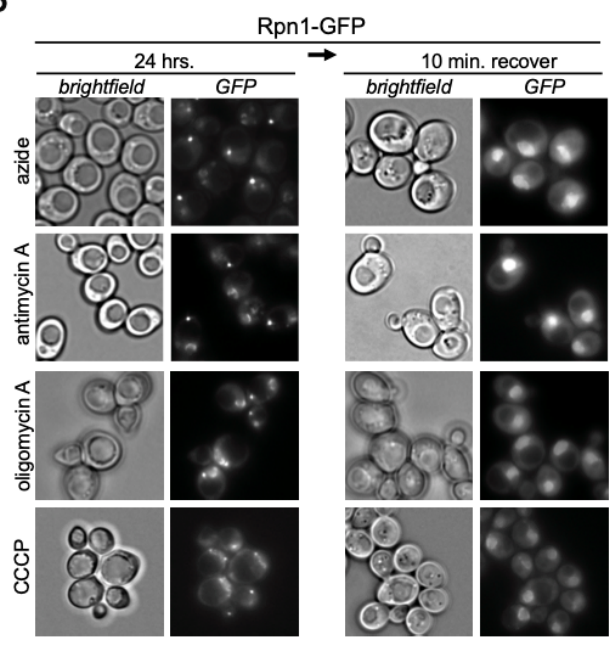

C
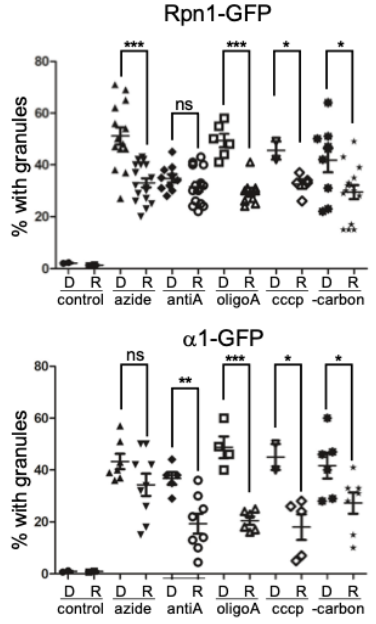

$E$
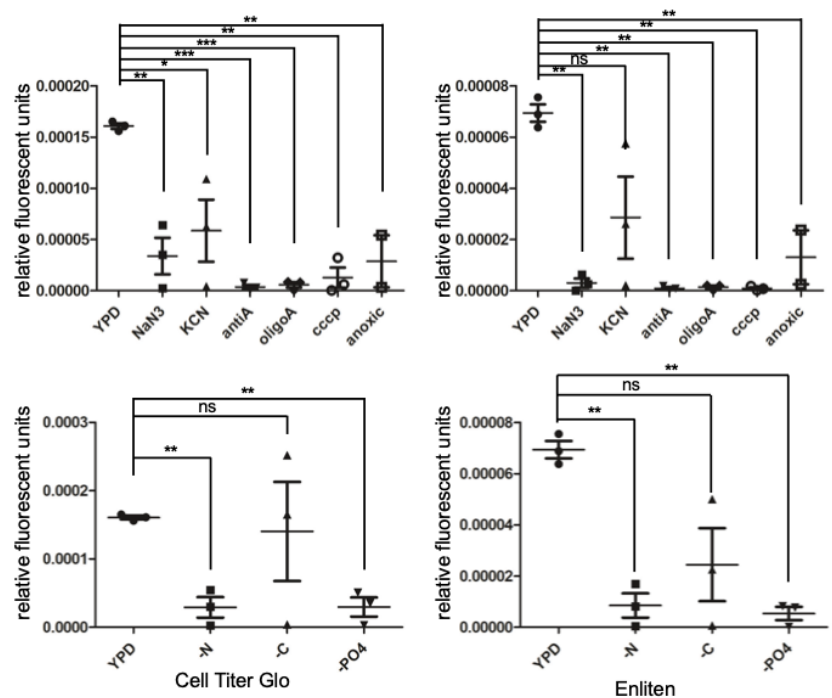
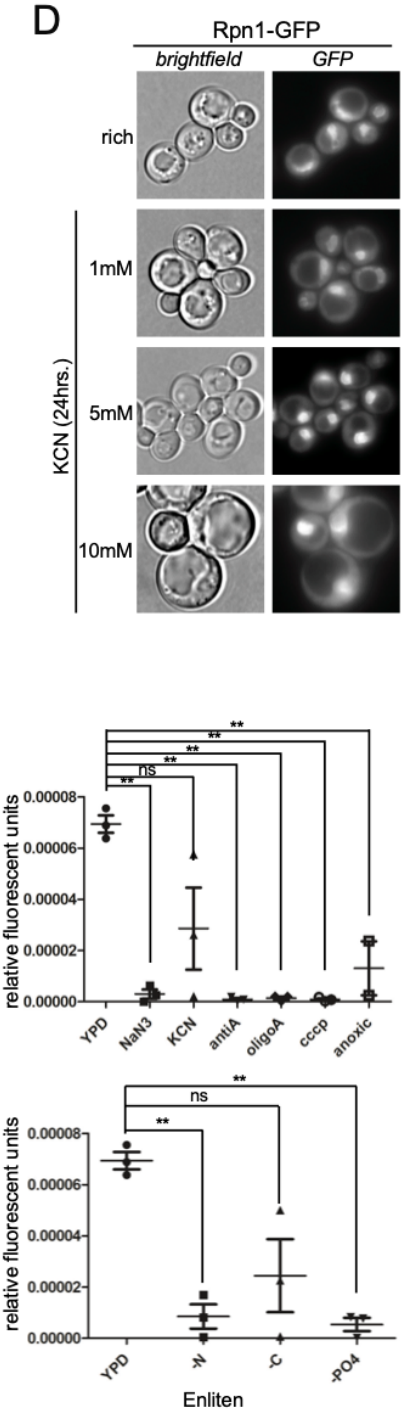

Figure 2. Mitochondrial inhibition induces proteasome granule formation. (A) Rpn1-GFP expressing cells were grown in rich media containing dextrose and treated with mitochondria inhibitors as described in materials and methods. Microscopy was performed 24 hours after inhibitor addition. Data are representative of three independent experiments. (B) Rpn1-GFP expressing cells treated for 24 hours with mitochondria inhibitors were washed and incubated in drug free media for 10 minutes. Data are representative of three independent experiments. (C) Rpn1-GFP or a1-GFP expressing cells were grown in media containing dextrose (D) or raffinose (R) for 4 hours, treated with mitochondria inhibitors, followed by and incubation for 24 hours. Microscopy was carried out and quantifications show the percentage of cells with granules. Unpaired t-tests were used to determine significance with $n>100$ for each datapoint. (D) Yeast expressing Rpn1-GFP were treated with increasing concentrations of potassium cyanide for 24 hours followed by microscopic analysis. Data are representative of three independent experiments. (E) ATP measurements were carried out following mitochondrial inhibition (upper panels) or nutrient starvation (lower panels) as described in materials and methods. Quantifications show relative ATP compared to untreated controls averaged from three independent experiments. Paired t-tests were used to evaluate significance. 
bioRxiv preprint doi: https://doi.org/10.1101/2022.01.13.476202; this version posted January 13, 2022. The copyright holder for this preprint (which was not certified by peer review) is the author/funder, who has granted bioRxiv a license to display the preprint in perpetuity. It is made available under aCC-BY-NC 4.0 International license.

A
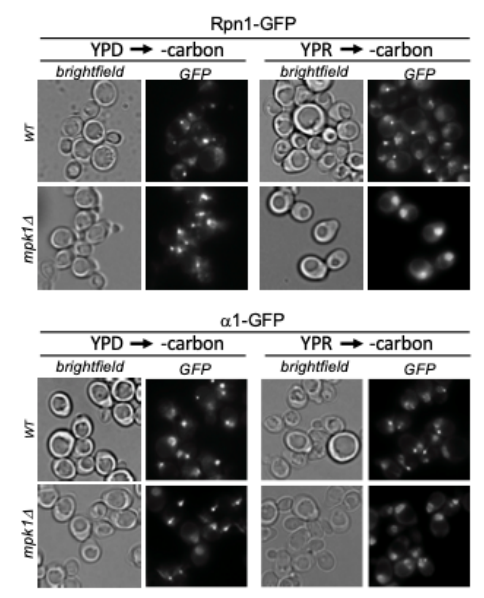

D
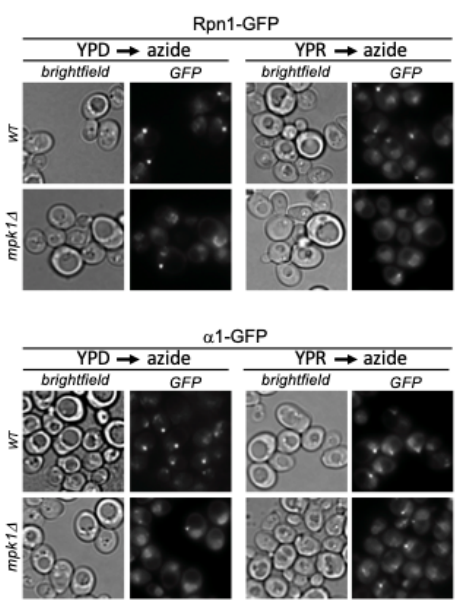

B
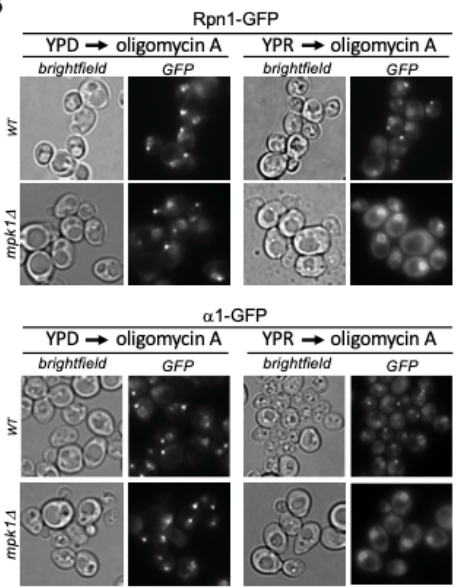

C
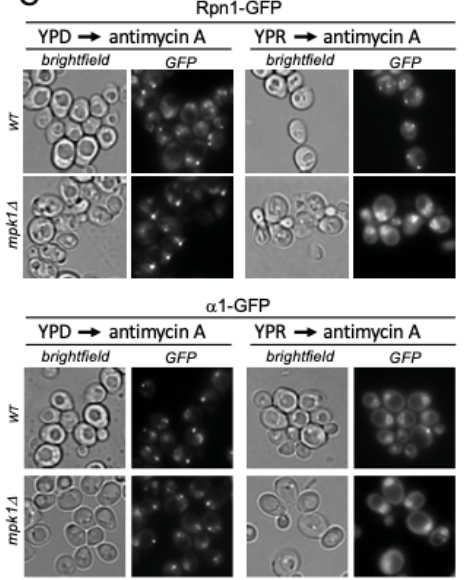
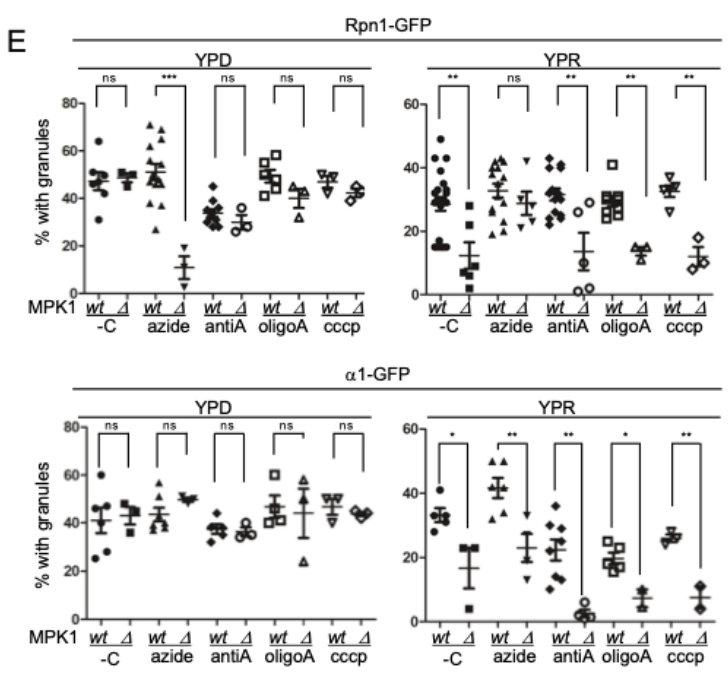

F

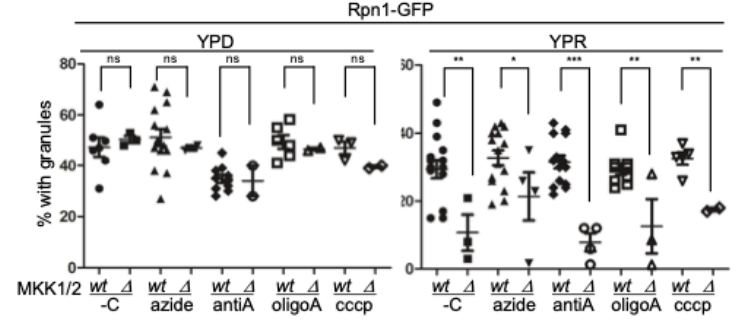

$\alpha 1-$ GFP

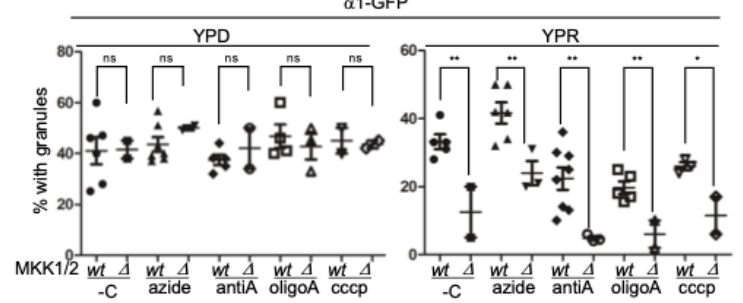

G

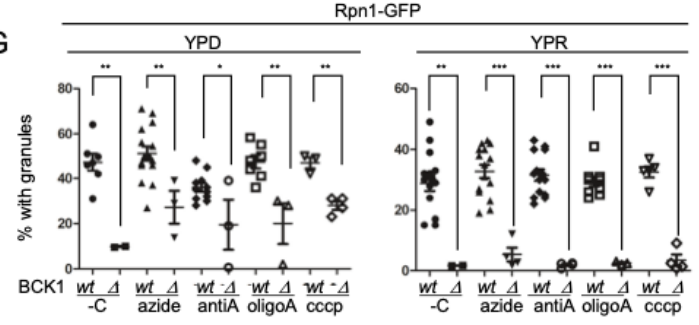

$\alpha 1-G F P$

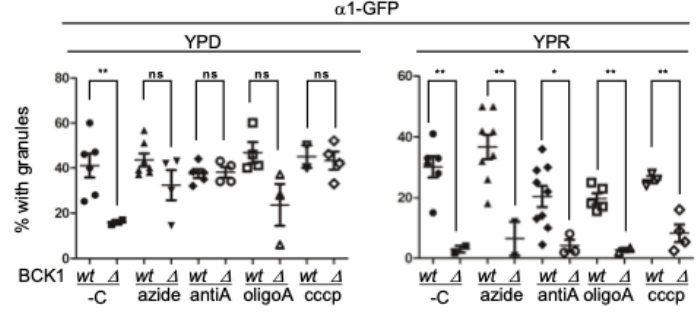


636 Figure 3. MAP kinase signaling is required for proteasome granule formation. (A-D) WT and $m p k 1 \Delta$ yeast

637 expressing Rpn1-GFP or $\alpha 1$-GFP were grown to log phase in rich media containing dextrose or raffinose. Next, cells

638 were starved for carbon, or treated with oligomycin A, antimycin A, or sodium azide for 24 hours and imaged. (E)

639 Quantification of the percent of granule formation from yeast cultured and treated as in A-D, or CCCP. Statistical

640 significance was determined using unpaired t-tests. Three or more independent experiments were quantified with

$641 \mathrm{n}>100$ for each datapoint. (F) wild type (wt) and MKK1 MKK2 double deletion yeast $(\Delta)$ expressing Rpn1-GFP or

$642 \alpha 1$-GFP were grown in dextrose or raffinose for four hours, starved for carbon or treated with mitochondria inhibitors

643 as above. Graphs show the percent of cells with granules after 24 hours with significance determined by unpaired t-

644 tests. At least three independent experiments were quantified with $n>100$ for each datapoint. (G) wild type and

$645 b c k 1 \Delta$ yeast expressing Rpn1-GFP or $\alpha 1$-GFP were grown to log phase in rich media containing dextrose or raffinose

646 then starved for carbon or treated with mitochondria inhibitors as above. Quantifications show the percent of cells that

647 formed granules after 24 hours. Unpaired t-tests were used to determine significance. At least three independent

648 experiments were quantified with $\mathrm{n}>100$ for each datapoint. 
A

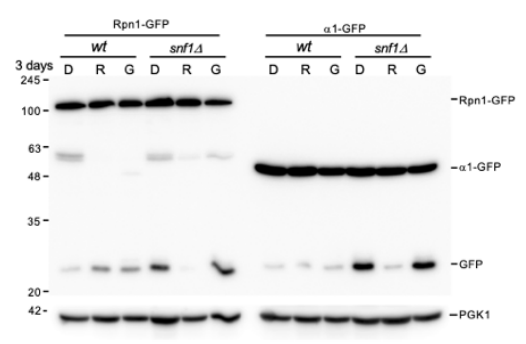

B

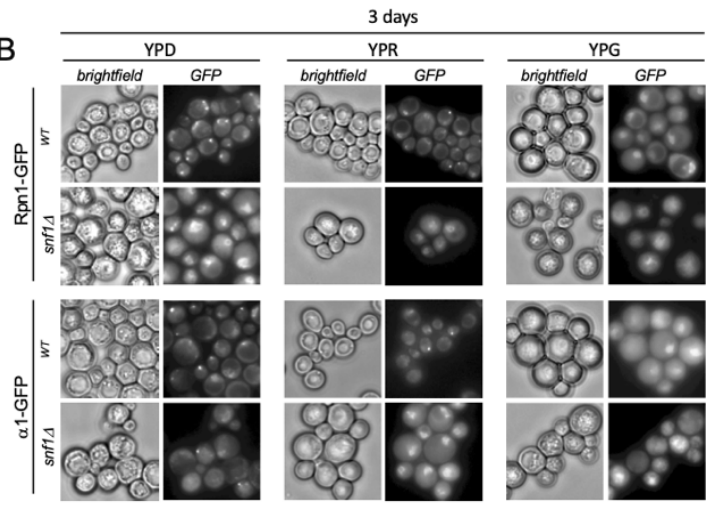

C
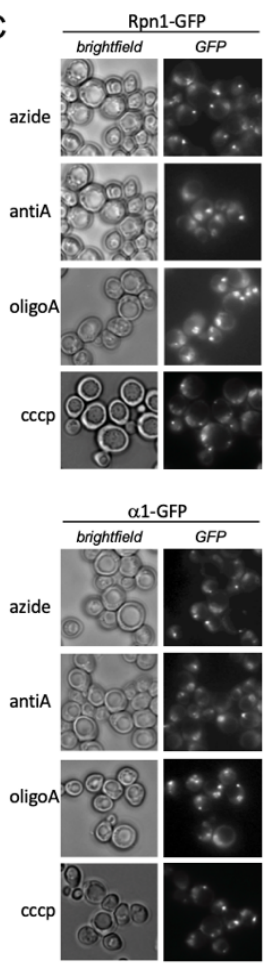
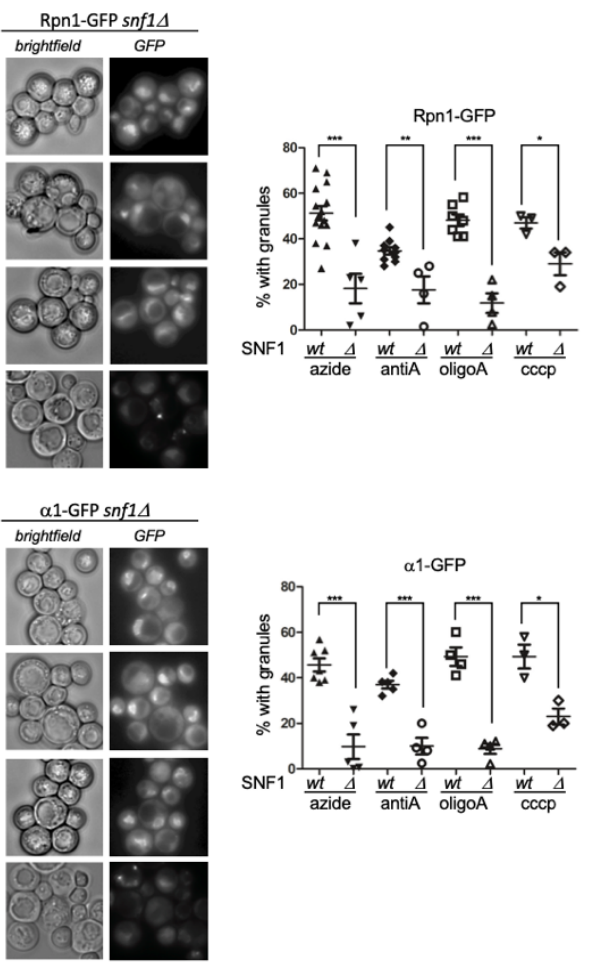

Figure 4. Snf1 is required for proteasome granule formation upon mitochondrial inhibition. (A) wild type (wt)

655 and SNF1 deleted yeast expressing Rpn1-GFP or $\alpha 1-G F P$ were grown for three days in rich media containing dextrose,

656 raffinose or glycerol (D, R and G respectively). Cells were harvested and lysed as described in materials and methods.

657 Immune blotting for GFP and Pgk1 was performed. Data are representative of two independent experiments. (B) Cells

658 from A were imaged microscopically to observe proteasome localization. Data are representative of two independent

659 experiments. (C) Yeast as in A and B were grown in YPD medium to log phase and starved for carbon or treated with

660 mitochondria inhibitors. Microscopy was performed and quantifications show the percent of cells that form granules

661 following mitochondria inhibition in the SNF1 deleted yeast compared to wild type. Significance was determined

662 using unpaired t-tests. At least three independent experiments with $\mathrm{n}>100$ for each datapoint, were used for

663 quantification. 

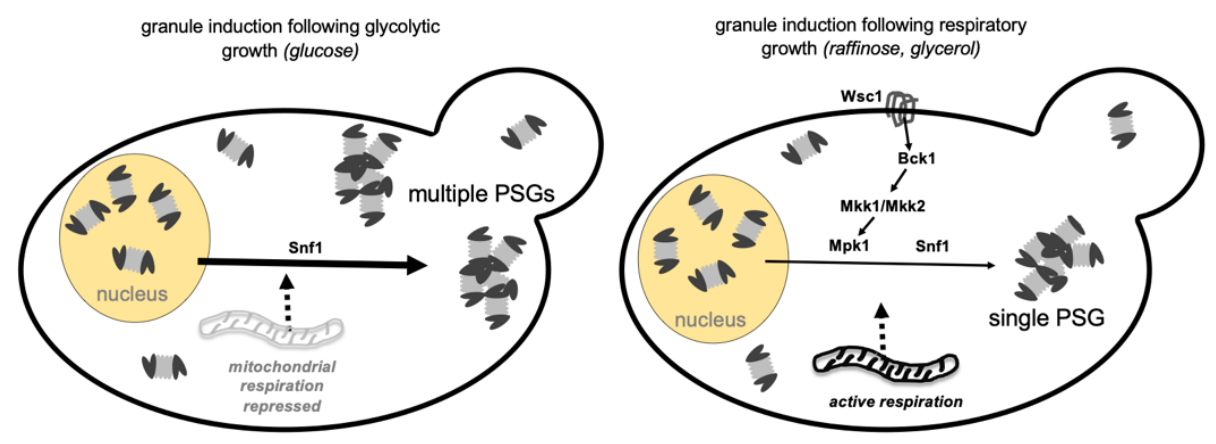

Figure 5. Model for PSG formation following glycolytic or respiratory growth

Switching from glycolytic growth media (i.e., with repressed mitochondrial respiration) to carbon starvation results in the formation of multiple proteasome granules per cell and more proteasome granules as compared to cells starved after growth in respiratory media. The kinase Snfl is required for proteasome granule formation upon mitochondrial inhibition but not carbon starvation. The cell integrity MAP kinase cascade (from Wsc1 to Mpk1) is required from proteasome granule formation following respiratory but not glycolytic growth.

\section{References}

Adachi, A., Koizumi, M. and Ohsumi, Y. (2017) 'Autophagy induction under carbon starvation conditions is negatively regulated by carbon catabolite repression.', The Journal of biological chemistry. American Society for Biochemistry and Molecular Biology, 292(48), pp. 19905-19918. doi: 10.1074/jbc.M117.817510.

682 Backhaus, K. et al. (2013) 'Mutations in SNF1 complex genes affect yeast cell wall strength', European Journal of Cell Biology. doi: 10.1016/j.ejcb.2014.01.001. BAO, W. et al. (2016) 'Induction of autophagy by the MG-132 proteasome inhibitor is associated with endoplasmic reticulum stress in MCF-7 cells', Molecular Medicine Reports, 13(1), pp. 796804. doi: 10.3892/mmr.2015.4599.

687 Boos, F. et al. (2019) 'Mitochondrial protein-induced stress triggers a global adaptive 688 transcriptional programme', Nature Cell Biology. doi: 10.1038/s41556-019-0294-5.

689 Bragoszewski, P. et al. (2013) 'The ubiquitin-proteasome system regulates mitochondrial 690 intermembrane space proteins.', Molecular and cellular biology. American Society for 
Microbiology (ASM), 33(11), pp. 2136-48. doi: 10.1128/MCB.01579-12.

692 Bragoszewski, P., Turek, M. and Chacinska, A. (2017) 'Control of mitochondrial biogenesis and

693 function by the ubiquitin-proteasome system.', Open biology. The Royal Society, 7(4). doi:

694 10.1098/rsob.170007.

695 Choi, W. H. et al. (2020) 'Aggresomal sequestration and STUB1-mediated ubiquitylation during mammalian proteaphagy of inhibited proteasomes', Proceedings of the National Academy of

697 Sciences of the United States of America. National Academy of Sciences, 117(32), pp. 19190698 19200. doi: 10.1073/pnas.1920327117.

699 Ciechanover, A. and Brundin, P. (2003) 'The Ubiquitin Proteasome System in Neurodegenerative 700 Diseases’, Neuron. Elsevier, 40(2), pp. 427-446. doi: 10.1016/S0896-6273(03)00606-8.

701 Cohen-Kaplan, V. et al. (2016) 'p62- and ubiquitin-dependent stress-induced autophagy of the mammalian 26S proteasome.', Proceedings of the National Academy of Sciences of the United States of America. National Academy of Sciences, 113(47), pp. E7490-E7499. doi: 10.1073/pnas.1615455113.

Deffieu, M. et al. (2013) 'Increased levels of reduced cytochrome b and mitophagy components are required to trigger nonspecific autophagy following induced mitochondrial dysfunction', Journal of Cell Science, 126(2), pp. 415-426. doi: 10.1242/jcs.103713.

708 van Deventer, S. et al. (2014) 'N-terminal acetylation and replicative age affect proteasome 709 localization and cell fitness during aging', Journal of Cell Science, 128(1), pp. 109-117. doi: $710 \quad 10.1242 /$ jcs.157354.

711 Dikic, I. (2017) 'Proteasomal and Autophagic Degradation Systems', Annual Review of

712 Biochemistry. Annual Reviews , 86(1), pp. 193-224. doi: 10.1146/annurev-biochem-061516713044908.

714 Enenkel, C. (2012) 'Using Native Gel Electrophoresis and Phosphofluoroimaging to Analyze

715 GFP-Tagged Proteasomes', in. Humana Press, pp. 339-348. doi: 10.1007/978-1-61779-474-2_23.

716 Enenkel, C. (2018) 'The paradox of proteasome granules', Current Genetics, 64(1), pp. 137-140.

717 doi: 10.1007/s00294-017-0739-y.

718 Feng, Y. et al. (2014) 'The machinery of macroautophagy.', Cell research. Shanghai Institutes for 719 Biological Sciences, Chinese Academy of Sciences, 24(1), pp. 24-41. doi: 10.1038/cr.2013.168. 720 Finley, D. (2009) 'Recognition and processing of ubiquitin-protein conjugates by the proteasome.', 721 Annual review of biochemistry, 78, pp. 477-513. doi: 
10.1146/annurev.biochem.78.081507.101607.

Finley, D. et al. (2012) 'The ubiquitin-proteasome system of Saccharomyces cerevisiae', Genetics, 192(October), pp. 319-360. doi: 10.1534/genetics.112.140467.

725

Finley, D., Özkaynak, E. and Varshavsky, A. (1987) 'The yeast polyubiquitin gene is essential for resistance to high temperatures, starvation, and other stresses', Cell. Cell Press, 48(6), pp. 10351046. doi: 10.1016/0092-8674(87)90711-2.

Fujii, K. et al. (2009) 'A role for ubiquitin in the clearance of nonfunctional rRNAs', Genes \& Development, 23(8), pp. 963-974. doi: 10.1101/gad.1775609.

730 Galdieri, L. et al. (2010) 'Transcriptional regulation in yeast during diauxic shift and stationary 731 phase.', Omics : a journal of integrative biology. Mary Ann Liebert, Inc., 14(6), pp. 629-38. doi: $732 \quad 10.1089 /$ omi.2010.0069.

733 Goebel, T. et al. (2020) 'Proteaphagy in mammalian cells can function independent of 734 ATG5/ATG7.', Molecular \& cellular proteomics: MCP. Mol Cell Proteomics. doi: 735 10.1074/mcp.RA120.001983.

736 Goldstein, A. L. and McCusker, J. H. (1999) 'Three new dominant drug resistance cassettes for 737 gene disruption in Saccharomyces cerevisiae.', Yeast (Chichester, England), 15(14), pp. 1541-53. doi: 10.1002/(SICI)1097-0061(199910)15:14<1541::AID-YEA476>3.0.CO;2-K.

739 Gribble, F. M. et al. (1997) 'Properties of cloned ATP-sensitive K+ currents expressed in Xenopus 740 oocytes', Journal of Physiology, 498(1). doi: 10.1113/jphysiol.1997.sp021843.

$741 \mathrm{Gu}, \mathrm{Z}$. C. et al. (2017) 'Ubiquitin orchestrates proteasome dynamics between proliferation and 742 quiescence in yeast.', Molecular biology of the cell. American Society for Cell Biology, 28(19), 743 pp. 2479-2491. doi: 10.1091/mbc.E17-03-0162.

744 Hailey, D. W., Davis, T. N. and Muller, E. G. D. (2002) 'Fluorescence resonance energy transfer 745 using color variants of green fluorescent protein.', Methods in enzymology, 351, pp. 34-49. 746 Available at: http://www.ncbi.nlm.nih.gov/pubmed/12073355 (Accessed: 12 June 2019).

747 Hanssum, A. et al. (2014) 'An Inducible Chaperone Adapts Proteasome Assembly to Stress', 748 Molecular Cell. Cell Press, 55(4), pp. 566-577. doi: 10.1016/J.MOLCEL.2014.06.017.

749 Hershko, A. and Ciechanover, A. (1998) 'The ubiquitin system.', Annual review of biochemistry. 750 Annual Reviews 4139 El Camino Way, P.O. Box 10139, Palo Alto, CA 94303-0139, USA, 67, 751 pp. 425-79. doi: 10.1146/annurev.biochem.67.1.425.

752 Heytler, P. G. and Prichard, W. W. (1962) 'A new class of uncoupling agents - Carbonyl cyanide 
753 phenylhydrazones', Biochemical and Biophysical Research Communications, 7(4). doi: 754 10.1016/0006-291X(62)90189-4.

755 Janke, C. et al. (2004) 'A versatile toolbox for PCR-based tagging of yeast genes: new fluorescent 756 proteins, more markers and promoter substitution cassettes', Yeast. John Wiley \& Sons, Ltd, 757 21(11), pp. 947-962. doi: 10.1002/yea.1142.

758 Junn, E. et al. (2002) 'Parkin accumulation in aggresomes due to proteasome impairment.', The 759 Journal of biological chemistry. American Society for Biochemistry and Molecular Biology, 760 277(49), pp. 47870-7. doi: 10.1074/jbc.M203159200.

761 Kabeya, Y. et al. (2000) 'LC3, a mammalian homologue of yeast Apg8p, is localized in 762 autophagosome membranes after processing.', The EMBO journal. European Molecular Biology 763 Organization, 19(21), pp. 5720-8. doi: 10.1093/emboj/19.21.5720.

764 Kamada, Y. et al. (2010) 'Tor Directly Controls the Atg1 Kinase Complex To Regulate 765 Autophagy', Molecular and Cellular Biology, 30(4), pp. 1049-1058. doi: 10.1128/MCB.0134476609.

767 Karmon, O. and Ben Aroya, S. (2020) 'Spatial Organization of Proteasome Aggregates in the 768 Regulation of Proteasome Homeostasis', Frontiers in Molecular Biosciences. doi: 769 10.3389/fmolb.2019.00150.

770 Kayikci, Ö. and Nielsen, J. (2015) ‘Glucose repression in Saccharomyces cerevisiae.', FEMS yeast 771 research. Oxford University Press, 15(6). doi: 10.1093/femsyr/fov068.

772 Kleijnen, M. F. et al. (2007) 'Stability of the proteasome can be regulated allosterically through 773 engagement of its proteolytic active sites', Nature Structural \& Molecular Biology, 14(12), pp.

774 1180-1188. doi: 10.1038/nsmb1335.

775 Ko, Y. H., Hong, S. and Pedersen, P. L. (1999) 'Chemical mechanism of ATP synthase. 776 Magnesium plays a pivotal role in formation of the transition state where ATP is synthesized from 777 ADP and inorganic phosphate.', The Journal of biological chemistry. American Society for 778 Biochemistry and Molecular Biology, 274(41), pp. 28853-6. doi: 10.1074/jbc.274.41.28853.

779 Kraft, C., Reggiori, F. and Peter, M. (2009) 'Selective types of autophagy in yeast', Biochimica et 780 Biophysica Acta (BBA) - Molecular Cell Research, 1793(9), pp. 1404-1412. doi: $781 \quad 10.1016 /$ j.bbamcr.2009.02.006.

782 Krause, S. A., Xu, H. and Gray, J. V. (2008) 'The synthetic genetic network around PKC1 783 identifies novel modulators and components of protein kinase $\mathrm{C}$ signaling in Saccharomyces 
784 cerevisiae', Eukaryotic Cell, 7(11). doi: 10.1128/EC.00222-08.

785 Kuranda, K. et al. (2006) 'Investigating the caffeine effects in the yeast Saccharomyces cerevisiae

786 brings new insights into the connection between TOR, PKC and Ras/cAMP signalling pathways',

787 Molecular Microbiology, 61(5), pp. 1147-1166. doi: 10.1111/j.1365-2958.2006.05300.x.

788 Kushnirov, V. V. (2000) 'Rapid and reliable protein extraction from yeast', Yeast. Wiley-

789 Blackwell, 16(9), pp. 857-860. doi: 10.1002/1097-0061(20000630)16:9<857::AID-

790 YEA561>3.0.CO;2-B.

791 Lang, M. J. et al. (2014) 'Glucose starvation inhibits autophagy via vacuolar hydrolysis and

792 induces plasma membrane internalization by down-regulating recycling', Journal of Biological

793 Chemistry, 289, pp. 16736-16747. doi: 10.1074/jbc.M113.525782.

794 Laporte, D. et al. (2008) 'Reversible cytoplasmic localization of the proteasome in quiescent yeast 795 cells', The Journal of Cell Biology, 181(5), pp. 737-745. doi: 10.1083/jcb.200711154.

796 Lavie, J. et al. (2018) 'Ubiquitin-Dependent Degradation of Mitochondrial Proteins Regulates

797 Energy Metabolism In Brief', CellReports, 23, pp. 2852-2863. doi: 10.1016/j.celrep.2018.05.013.

798 Levin, D. E. (2005) ' Cell Wall Integrity Signaling in Saccharomyces cerevisiae ', Microbiology

799 and Molecular Biology Reviews, 69(2). doi: 10.1128/mmbr.69.2.262-291.2005.

800 Li, D. et al. (2015) 'A fluorescent tool set for yeast Atg proteins', Autophagy, 11(6), pp. 954-960.

801 doi: 10.1080/15548627.2015.1040971.

$802 \mathrm{Li}$, J. et al. (2019) 'AMPK regulates ESCRT-dependent microautophagy of proteasomes

803 concomitant with proteasome storage granule assembly during glucose starvation', PLOS

804 Genetics. Edited by A. K. Hopper, 15(11), p. e1008387. doi: 10.1371/journal.pgen.1008387.

$805 \mathrm{Li}, \mathrm{W}$. et al. (2008) 'Genome-wide and functional annotation of human E3 ubiquitin ligases

806 identifies MULAN, a mitochondrial E3 that regulates the organelle's dynamics and signaling',

807 PLoS ONE, 3(1). doi: 10.1371/journal.pone.0001487.

808 Mao, K. et al. (2011) 'Two MAPK-signaling pathways are required for mitophagy in

809 Saccharomyces cerevisiae', The Journal of Cell Biology, 193(4), pp. 755-767. doi: $810 \quad 10.1083 / \mathrm{jcb} .201102092$.

811 Marshall, R. S. et al. (2015) 'Autophagic Degradation of the 26S Proteasome Is Mediated by the 812 Dual ATG8/Ubiquitin Receptor RPN10 in Arabidopsis', Molecular Cell, 58, pp. 1053-1066. doi: 813 10.1016/j.molcel.2015.04.023.

814 Marshall, R. S., McLoughlin, F. and Vierstra, R. D. (2016) 'Autophagic Turnover of Inactive 26S 
815 Proteasomes in Yeast Is Directed by the Ubiquitin Receptor Cue5 and the Hsp42 Chaperone', Cell 816 Reports. Cell Press, 16(6), pp. 1717-1732. doi: 10.1016/J.CELREP.2016.07.015.

817 Marshall, R. S. and Vierstra, R. D. (2018) 'Proteasome storage granules protect proteasomes from 818 autophagic degradation upon carbon starvation', eLife, 7. doi: 10.7554/eLife.34532.

819 May, A. I., Prescott, M. and Ohsumi, Y. (2020) 'Autophagy facilitates adaptation of budding yeast

820 to respiratory growth by recycling serine for one-carbon metabolism', Nature Communications,

821 11(1). doi: 10.1038/s41467-020-18805-x.

822 Mochida, K. et al. (2015) 'Receptor-mediated selective autophagy degrades the endoplasmic

823 reticulum and the nucleus', Nature, 522(7556), pp. 359-362. doi: 10.1038/nature14506.

824 Nemec, A. A. et al. (2017) 'Autophagic clearance of proteasomes in yeast requires the conserved

825 sorting nexin Snx4.', The Journal of biological chemistry. American Society for Biochemistry and

826 Molecular Biology, 292(52), pp. 21466-21480. doi: 10.1074/jbc.M117.817999.

827 Nicastro, R. et al. (2015) 'Enhanced amino acid utilization sustains growth of cells lacking 828 Snfl/AMPK', Biochimica et Biophysica Acta - Molecular Cell Research, 1853(7). doi: $829 \quad 10.1016 /$ j.bbamcr.2015.03.014.

830 Ogata, M. et al. (2006) 'Autophagy is activated for cell survival after endoplasmic reticulum 831 stress.', Molecular and cellular biology. American Society for Microbiology (ASM), 26(24), pp. 832 9220-31. doi: 10.1128/MCB.01453-06.

833 Peters, L. Z. et al. (2016) 'Proteasome storage granules are transiently associated with the insoluble 834 protein deposit in Saccharomyces cerevisiae', Journal of Cell Science, 129(6), pp. 1190-1197. 835 doi: $10.1242 /$ jcs.179648.

836 Peters Lee Zeev, Z. et al. (2013) 'Formation and dissociation of proteasome storage granules are 837 regulated by cytosolic pH', Journal of Cell Biology, 201(5), pp. 663-671. doi: $838 \quad 10.1083 /$ jcb.201211146.

839 Petti, A. A. et al. (2011) 'Survival of starving yeast is correlated with oxidative stress response 840 and nonrespiratory mitochondrial function', Proceedings of the National Academy of Sciences, 841 108(45), pp. E1089-E1098. doi: 10.1073/pnas.1101494108.

842 Rittinger, K. and Ikeda, F. (2017) 'Linear ubiquitin chains: enzymes, mechanisms and biology', 843 Open Biology, 7(4), p. 170026. doi: 10.1098/rsob.170026.

844 Rousseau, A. and Bertolotti, A. (2016) 'An evolutionarily conserved pathway controls proteasome 845 homeostasis.', Nature. Nature Publishing Group, 536(7615), pp. 184-189. doi: 
847 Schmidt, R. M. et al. (2019) 'The proteasome biogenesis regulator Rpn4 cooperates with the 848 unfolded protein response to promote ER stress resistance', eLife, 8. doi: 10.7554/eLife.43244.

849 Schrader, E. K., Harstad, K. G. and Matouschek, A. (2009) 'Targeting proteins for degradation.', 850 Nature chemical biology, 5(11), pp. 815-22. doi: 10.1038/nchembio.250.

851 Schüller, H. J. (2003) 'Transcriptional control of nonfermentative metabolism in the yeast

852 Saccharomyces cerevisiae', Current Genetics. doi: 10.1007/s00294-003-0381-8.

853 Secco, D. et al. (2012) 'Phosphate homeostasis in the yeast Saccharomyces cerevisiae, the key role 854 of the SPX domain-containing proteins', FEBS Letters. No longer published by Elsevier, 586(4), 855 pp. 289-295. doi: 10.1016/J.FEBSLET.2012.01.036.

856 Shaid, S. et al. (2013) 'Ubiquitination and selective autophagy.', Cell death and differentiation.

857 Nature Publishing Group, 20(1), pp. 21-30. doi: 10.1038/cdd.2012.72.

858 Sundin, B. A. et al. (2004) 'Localization of proteins that are coordinately expressed with Cln2 859 during the cell cycle', Yeast. John Wiley \& Sons, Ltd, 21(9), pp. 793-800. doi: 10.1002/yea.1133.

860 Symersky, J. et al. (2012) 'Oligomycin frames a common drug-binding site in the ATP synthase', 861 Proceedings of the National Academy of Sciences of the United States of America, 109(35). doi: $86210.1073 /$ pnas.1207912109.

863 Takeshige, K. et al. (1992) 'Autophagy in yeast demonstrated with proteinase-deficient mutants 864 and conditions for its induction', The Journal of Cell Biology, 119(2), pp. 301-311. doi: $86510.1083 /$ jcb.119.2.301.

866 Turcotte, B. et al. (2010) 'Transcriptional regulation of nonfermentable carbon utilization in 867 budding yeast', FEMS Yeast Research. doi: 10.1111/j.1567-1364.2009.00555.x.

868 Um, J. W. et al. (2010) 'Parkin directly modulates 26S proteasome activity.', The Journal of 869 neuroscience: the official journal of the Society for Neuroscience. Society for Neuroscience, 870 30(35), pp. 11805-14. doi: 10.1523/JNEUROSCI.2862-09.2010.

871 Uriarte, M. et al. (2021) 'Starvation-induced proteasome assemblies in the nucleus link amino acid 872 supply to apoptosis', Nature Communications, 12(1). doi: 10.1038/s41467-021-27306-4.

873 Verna, J. et al. (1997) 'A family of genes required for maintenance of cell wall integrity and for 874 the stress response in Saccharomyces cerevisiae', Proceedings of the National Academy of 875 Sciences of the United States of America, 94(25). doi: 10.1073/pnas.94.25.13804.

876 Waite, K. A. et al. (2015) 'Starvation Induces Proteasome Autophagy with Different Pathways for 
877 Core and Regulatory Particle.', The Journal of biological chemistry, p. M115.699124-. doi: $878 \quad 10.1074 /$ jbc.M115.699124.

879 Waite, K. A. et al. (2016) 'Starvation induces proteasome autophagy with different pathways for 880 core and regulatory particles', Journal of Biological Chemistry, 291(7), pp. 3239-3253. doi: $881 \quad 10.1074 /$ jbc.M115.699124.

882 Waite, K. A. et al. (2021) 'Proteaphagy is specifically regulated and requires factors dispensable 883 for general autophagy', Journal of Biological Chemistry, 298(1), p. 101494. doi: $884 \quad 10.1016 /$ j.jbc.2021.101494.

885 Waite, K. A., Burris, A. and Roelofs, J. (2020) 'Tagging the proteasome active site $\beta 5$ causes tag 886 specific phenotypes in yeast', Scientific Reports, 10(1). doi: 10.1038/s41598-020-75126-1.

887 Webb, J. L. et al. (2003) ' $\alpha$-Synuclein Is Degraded by Both Autophagy and the Proteasome', 888 Journal of Biological Chemistry, 278(27), pp. 25009-25013. doi: 10.1074/jbc.M300227200.

889 Weberruss, M. H. et al. (2013) 'Blm10 facilitates nuclear import of proteasome core particles.', 890 The EMBO journal. European Molecular Biology Organization, 32(20), pp. 2697-707. doi: 891 10.1038/emboj.2013.192.

892 Wikström, M. K. F. and Berden, J. A. (1972) 'Oxidoreduction of cytochrome b in the presence of 893 antimycin', BBA - Bioenergetics, 283(3). doi: 10.1016/0005-2728(72)90258-7.

894 Willis, S. D. et al. (2018) 'Snfl cooperates with the CWI MAPK pathway to mediate the 895 degradation of Med13 following oxidative stress', Microbial Cell. doi: 10.15698/mic2018.08.641.

896 Yasuda, S. et al. (2020) 'Stress- and ubiquitylation-dependent phase separation of the proteasome', 897 Nature, 578(7794). doi: 10.1038/s41586-020-1982-9.

898 Yi, C. et al. (2017) 'Formation of a Snf1-Mec1-Atg1 Module on Mitochondria Governs Energy 899 Deprivation-Induced Autophagy by Regulating Mitochondrial Respiration', Developmental Cell, 900 41(1), pp. 59-71.e4. doi: 10.1016/j.devcel.2017.03.007. 